\title{
Effects of probiotics on chemotherapy in patients with lung cancer
}

\author{
YANG TIAN ${ }^{1}$, MING LI $^{2}$, WEI SONG ${ }^{1}$, RUI JIANG ${ }^{1}$ and YAN QING LI ${ }^{2}$ \\ ${ }^{1}$ Department of Oncology, Shandong Provincial Hospital Affiliated to Shandong University; \\ ${ }^{2}$ Department of Gastroenterology, Shandong University, Qilu Hospital, Jinan, Shandong 250012, P.R. China
}

Received March 30, 2018; Accepted November 21, 2018

DOI: $10.3892 / \mathrm{ol} .2019 .9906$

\begin{abstract}
Chemotherapy damages the intestinal mucosa, causing adverse gastrointestinal reactions. Clostridium butyricum (C. butyricum) reduces the incidence of diarrhea in digestive diseases, including inflammatory bowel disease. Therefore, the aim of the present study was to investigate the role of C. butyricum in patients undergoing chemotherapy. A total of 41 participants with lung cancer were enrolled, and divided into the $C$. butyricum (CB) or placebo group using 1:1 randomization to obtain $20 \mathrm{CB}$ and 21 placebo participants. On the first and last day of the 3-week intervention, blood and stool samples were collected and analyzed. To analyze stool flora, 16S ribosomal RNA sequencing was performed. The incidence of chemotherapy-induced diarrhea was lower in the CB group compared with the placebo group. The lymphocyte count and platelet/lymphocyte ratio (PLR) was markedly altered between the two groups. Neutrophil/lymphocyte ratio (NLR) and PLR decreased within the CB group. At week 3, the lymphocyte/monocyte ratio (LMR) was higher in the $\mathrm{CB}$ group compared with the placebo group. Alterations in lymphocyte subsets and immunoglobulin levels were not significantly different. Albumin (ALB) level and weight did not differ significantly between the two groups. At 3 weeks the total flora diversity did not decrease in either group. Phyla in the CB group varied slightly, while the proportion of Firmicutes in the placebo group decreased significantly. No statistically significant difference was observed between the two groups, though the genera producing short-chain fatty acids tended to increase, and the pathogenic genera tended to decrease in the $\mathrm{CB}$ group, which was almost the opposite of the observation in the placebo group. Operational taxonomy unit analysis revealed a notable increase in beneficial flora, including the Clostridium and Lactobacillus genera of the CB group, compared with the placebo group. The present study highlighted that $C$. butyricum reduced chemotherapy-induced diarrhea in patients with lung
\end{abstract}

Correspondence to: Dr Yan Qing Li, Department of Gastroenterology, Shandong University, Qilu Hospital, 107 Wenhuaxi Road, Jinan, Shandong 250012, P.R. China

E-mail: liyanqing@sdu.edu.cn

Key words: intestinal microflora, probiotic, chemotherapy, systemic inflammatory response cancer, reduced the systemic inflammatory response system and encouraged homeostatic maintenance.

\section{Introduction}

Lung cancer is the most frequently occurring cancer and the leading cause of cancer-associated mortality in China (1). Lung cancer involves malignant proliferation, invasion and metastasis (2). Surgery is a possible cure for early stage lung cancer (3); however, the majority of tumors are already in advanced stages when discovered $(4,5)$. Despite the emergence of new treatments, including targeted therapies and immunotherapy, certain patients with advanced or recurrent lung cancer require systemic chemotherapy (6). However, systemic chemotherapy commonly results in side effects including nausea, vomiting and diarrhea $(7,8)$. These side effects may worsen the patient's nutritional status, reduce immune function, delay the treatment cycle and increase the treatment costs (9-11). Among these effects, chemotherapy-induced diarrhea may be caused by intestinal epithelial cell apoptosis, intestinal barrier dysfunction, alterations to the intestinal flora and proinflammatory cytokine production (12).

Studies on intestinal flora have identified certain bacteria that are beneficial to the body, which when ingested in sufficient quantities can exhibit a positive effect on the host and serve as a probiotic (13). Probiotics may help to repair the intestinal barrier, alleviate gastrointestinal inflammation and maintain gut homeostasis; more specifically, Clostridium butyricum (C. butyricum) has been reported to regulate the gut homeostasis, reduce inflammation and reduce the incidence of diarrhea in digestive diseases including inflammatory bowel disease (IBD) $(14,15)$, and has been used to treat gastrointestinal disorders, including IBD and antibiotic-associated diarrhea $(16,17)$.

Therefore, it is speculated that the use of probiotics, including $C$. butyricum, may reduce chemotherapy-induced diarrhea (CID). However, there is currently not sufficient and clear clinical evidence to support the safety and efficacy of any probiotics, including the use of $C$. butyricum in CID $(18,19)$. The present study adopted the design of a prospective clinical trial to study the role of $C$. butyricum in patients with lung cancer undergoing chemotherapy. In this trial, blood samples were collected and analyzed to evaluate inflammatory, immune and nutritional indicators. The fetal flora were analyzed using the $16 \mathrm{~S}$ ribosomal RNA (rRNA) sequencing technique, and the association between probiotic use, the inflammatory response, 
immunity and nutrition was investigated, to provide supporting evidence for the use of probiotics in clinical practice.

\section{Materials and methods}

Patients. Patients with lung cancer who were admitted to the Shandong Provincial Hospital (Jinan, China) for chemotherapy were recruited between July 2015 and June 2016. The inclusion criteria were as follows: i) Between 18 and 80 years old; ii) diagnosed with lung cancer as the first primary tumor. The exclusion criteria were as follows: i) Severe systemic diseases including heart, liver or kidney dysfunction; ii) previous chemotherapy or other anti-cancer therapies; iii) probiotic, prebiotic or antibiotic therapy during the 2 months prior to recruitment; iv) allergy to micro ecological agents; v) pregnant or breast feeding women; vi) coagulopathy; and vii) the inability to provide informed consent. All participants provided written informed consent. The protocol was approved by the Institutional Ethics Committee of Shandong Provincial Hospital and registered at ClinicalTrials.gov with the identifier NCT02771470. The registration date was June 14th, 2015. The procedures were conducted in accordance with the approved guidelines.

Study design. The present study was prospective, random, double blind and placebo comparative; the work flow is illustrated in Fig. 1. A total of 50 patients were recruited and assessed for eligibility. Five patients were excluded as they did not meet the inclusion criteria $(n=1)$, met the exclusion criteria $(n=2)$ or for another reason $(n=2)$. And then forty-five patients were randomly placed at a 1:1 ratio in the $C$. butyricum group (CB) or the placebo group using a random number table $(n=23$ in CB group, $n=22$ in placebo group). Ultimately, 41 patients were included in the final cohort. Patients were subjected to platinum-based combination chemotherapy every 3 weeks according to the National Comprehensive Cancer Network guidelines (20). Patients were administered treatment at two time points; the day preceding the first course of chemotherapy (baseline) and the day preceding the second course (week three). From the baseline day, three C. butyricum (420 mg/tablet) or placebo tablets (Qingdao East China Sea Pharmaceutical Co., Ltd, Qingdao, China) were administered three times per day, for 3 weeks. The treatments were administered by assistants who were not aware of whether the drugs were placebo or not. Blood and stool samples were collected at the two time points.

Record of adverse effects. Adverse effects, including nausea, vomiting and diarrhea were assessed and graded according to the Criteria for Adverse Events, version 4 (21). Nausea is a disorder characterized by a queasy sensation and/or the urge to vomit. The grade of nausea was divided into three levels as follows: i) Loss of appetite without alteration in eating habits; ii) decreased oral intake without significant weight loss, dehydration or malnutrition; or iii) inadequate oral caloric food or fluid intake, tube feeding, total parenteral nutrition, or hospitalization indicated. Vomiting is a disorder characterized by the reflexive act of ejecting the contents of the stomach through the mouth. The grade of vomiting was divided into five levels as follow: i) One to two episodes within $24 \mathrm{~h}$; ii) three to five
Table I. Patient characteristics of the $\mathrm{CB}$ and placebo groups.

\begin{tabular}{lccc}
\hline Characteristic & CB group & Placebo group & P-value \\
\hline Sex & & & \\
$\quad$ Male & 15 & 15 & 0.837 \\
Female & 5 & 6 & \\
Age, years & $57 \pm 8.75$ & $54 \pm 8.35$ & 0.196 \\
Cancer stage & & & \\
I & 0 & 0 & 0.986 \\
II & 3 & 3 & \\
III & 8 & 8 & \\
IV & 9 & 10 & \\
\hline
\end{tabular}

Results are expressed as the median \pm standard deviation. Statistical significance was determined by Wilcoxon test. CB, Clostridium butyricum.

episodes within $24 \mathrm{~h}$; iii) more than six episodes within $24 \mathrm{~h}$, tube feeding, total parenteral nutrition or hospitalization indicated; iv) life-threatening consequences, urgent intervention indicated; or v) mortality. Diarrhea is a disorder characterized by frequent and watery bowel movements, and the grade of diarrhea was divided into five levels as follows: i) An increase in stool evacuation per day of less than four times above baseline; ii) an increase in stool evacuation per day of four to six times above baseline; iii) an increase in stool evacuation per day of more than seven times above baseline, incontinence and hospitalization indicated; iv) life-threatening consequences, urgent intervention indicated; or v) mortality. The grade scores were evaluated and recorded.

Blood testing. Morning fasting venous blood was taken for routine blood testing with the XN-9000 automatic hematology analyzer (Sysmex Corporation, Kobe, Japan) and albumin evaluation with the Olympus AU5800 biochemical analyzer (Beckman Coulter, Inc., Brea, CA, USA).

Lymphocyte subsets including $\mathrm{CD}^{+} \mathrm{T}$ lymphocytes, $\mathrm{CD}^{+} \mathrm{T}$ lymphocytes, natural killer $(\mathrm{NK})$ cells and $\mathrm{CD} 19^{+} \mathrm{B}$ lymphocytes were quantified by flow cytometry. First, $2 \mathrm{ml}$ of fasting blood was extracted and placed in EDTA-K2 anticoagulant tube, where it was mixed well and stored at room temperature $\left(25^{\circ} \mathrm{C}\right)$ for $<24 \mathrm{~h}$. A $12 \times 75 \mathrm{~mm}$ Falcon tube was then marked with the sample number. Each blood sample was added to two tubes containing two different types of mixed antibody reagents included in the BD MultiTEST IMK kit (catalog no. 340505; Becton, Dickinson and Company, Franklin Lakes, NJ, USA). One was the BD Multitest CD3/CD8/CD45/CD4 mixed antibody reagent, including the following antibodies: Anti-CD3-FITC, anti-CD8-PE, anti-CD45-PerCP and anti-CD4-APC. The other reagent was the BD Multitest CD3/CD16CD56/CD45/CD19 mixed antibody reagent, including the following antibodies: Anti-CD3-FITC, anti-CD16/56-PE, anti-CD45-PerCP and anti-CD19-APC. Each mixed antibody reagent contained $20 \mu \mathrm{l}$ and was provided in $1 \mathrm{ml}$ of buffered saline with $0.1 \%$ sodium azide. Subsequently, $50 \mu 1$ of each blood sample was added to the bottom of the sample tubes. The antibody was 
Flow

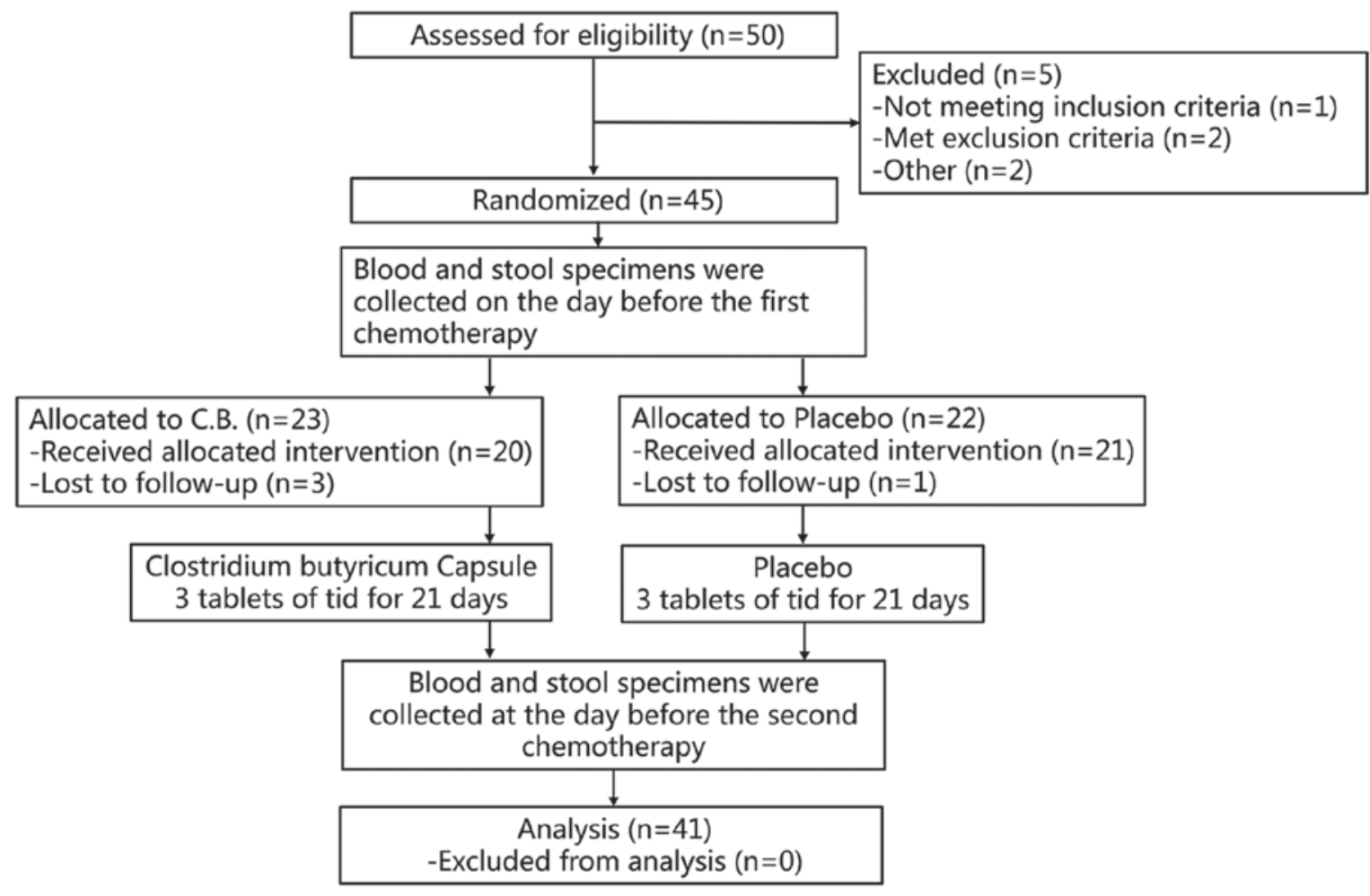

Figure 1. Flow chart of study. Patients with lung cancer were randomly divided at a 1:1 ratio to receive $\mathrm{CB}$ or the placebo treatment. Blood and stool specimens were collected at baseline. From the baseline onward, CB or the placebo was administered at a dose of three tablets/day, three times per day, for a total of 3 weeks. After 3 weeks, blood and stool samples were collected again. CB, Clostridium butyricum.

then mixed with the blood sample at low speed for $3 \mathrm{sec}$ and incubated at room temperature for $15 \mathrm{~min}$ in the dark. Following the incubation, $450 \mu 1$ of $1 \mathrm{x}$ FACS hemolysin (Becton, Dickinson and Company) was added to each tube. The sample mixed with antibody reagent 1 was incubated at room temperature for $15 \mathrm{~min}$ in the dark. Simultaneously, the sample mixed with antibody reagent 2 was incubated at room temperature for $10 \mathrm{~min}$ in the dark. The samples were then measured using a flow cytometer and analyzed automatically by FACS Canto Clinical software version 2.4 (FACS Canto II; Becton, Dickinson and Company). Samples positive for CD4-APC, CD3-FITC and CD45-PerCP were classified as CD4+ T lymphocytes. Samples positive for CD8-PE, CD3-FITC and CD45-PerCP positive were CD8+ T lymphocytes. Samples positive for CD16/56-PE, CD3-FITC and CD45-PerCP positive are NK cells. CD19-APC, CD3-FITC and $\mathrm{CD} 45$-PerCP were determine to be $\mathrm{B}$ lymphocytes.

Immunoglobulins (Igs) were measured by the scattering turbidimetric method (22) using the Siemens BNII specific protein analyzer (Siemens AG, Munich, Germany). The following inflammatory response biomarker ratios were evaluated: Neutrophil lymphocyte ratio (NLR), lymphocyte/monocyte ratio (LMR) and platelet/lymphocyte ratio (PLR).

\section{Stool specimens test methods}

DNA extraction and sequencing. Stool samples $(\sim 2-4 \mathrm{~g})$ were placed into sterile tubes, and frozen at $-20^{\circ} \mathrm{C}$ within $5 \mathrm{~min}$, and subsequently stored at $-80^{\circ} \mathrm{C}$ within $24 \mathrm{~h}$. The total DNA was extracted, amplified and sequenced by Shanghai Majorbio Pharmaceutical Technology Co., Ltd. (Shanghai, China) according to the standardized protocol 19. The V3-V4 region of the $16 \mathrm{~S}$ ribosomal subunit gene was amplified using $338 \mathrm{~F} / 806 \mathrm{R}$ barcoded primers (338F, 5'-ACTCCTACGGGAGGCAGC AG-3' and 806R, 5'-GGACTACHVGGGTWTCTAAT-3') and sequenced using the Illumina Miseq PE300 platform (Shanghai Majorbio Pharmaceutical Technology Co., Ltd.).

Raw sequencing data processing. Sequencing data was obtained using Mothur Software v 1.33.0 (https://www.mothur. org/). The raw sff files were decoded, denoised, trimmed and aligned to Silva references (Release 119) (23) using the default parameters. The sequences were clustered with the same operational taxonomy unit (OTU) if their distances were $<0.03$. Taxonomy was assigned to each OTU using the classify. otu command and further represented by the finest taxonomic name. The OTU table was converted to biom files, and the taxa relative abundances at domain to genus levels were generated using the summarize_taxa.py command in QIIME v1.8.0 (http://qiime.org/).

Community diversity analysis and significance test for quantity differences evaluation. The community diversity was evaluated prior to conducting the significant test for quantity difference evaluation between groups, using the Illumina Miseq PE300 platform (Shanghai Majorbio Pharmaceutical Technology Co., Ltd, Shanghai, China). The Shannon index and Chao index were calculated to indicate community 
Table II. Variations in weight and ALB of the CB and placebo groups.

\begin{tabular}{|c|c|c|c|c|c|}
\hline \multirow[b]{2}{*}{ Variable } & \multirow[b]{2}{*}{ Group } & \multicolumn{2}{|c|}{ Time point } & \multirow[b]{2}{*}{ P-intra value } & \multirow[b]{2}{*}{ P-value } \\
\hline & & Baseline & Week3 & & \\
\hline \multirow[t]{3}{*}{ ALB (g/l) } & CB & $38.28 \pm 3.928$ & $39.135 \pm 4.611$ & 0.4987 & \multirow[t]{3}{*}{0.1589} \\
\hline & Placebo & $38.49 \pm 4.767$ & $37.32 \pm 5.047$ & 0.3519 & \\
\hline & P-inter value & 0.4414 & 0.3751 & - & \\
\hline \multirow[t]{3}{*}{ Weight (kg) } & $\mathrm{CB}$ & $65.5 \pm 9.13$ & $65.47 \pm 8.55$ & 0.9460 & \multirow[t]{3}{*}{0.501} \\
\hline & Placebo & $64.5 \pm 11.67$ & $63.78 \pm 11.80$ & 0.7626 & \\
\hline & P-inter value & 0.5925 & 0.4810 & - & \\
\hline
\end{tabular}

Results are expressed as the median \pm standard deviation. The P-intra value indicates the P-value between two time points within the same group, calculated by Wilcoxon test. The P-inter value indicates the P-value between the two groups at the same time point, calculated by Wilcoxon test. P-values were obtained with the comparison of the changes of two groups calculated using the Wilcoxon test. ALB, albumin level; CB, Clostridium butyricum.

diversity and richness. Principal coordinates analysis (PCoA) is a visualization method to evaluate the similarity or variability of data. Analysis of similarities (ANOSIM) serves to test whether differences between groups are significantly greater than intra-group differences to determine whether grouping makes sense. Adonis analysis analyzes the different grouping factors to explain the differences between samples. Species diversity was tested in different microbial community groups to assess the significance of the observed differences based on the obtained community abundance data.

Statistical analysis. The Wilcoxon test was used to compare the difference of each index and the difference of changes of each index of two groups using R 3.4.3 (https://www.r-project. org/). The Kruskal-Wallis test with Dunn multiple comparisons test was used to analyze the difference of OTUs between two groups. Community diversity analysis and significant differences were analyzed using the Illumina Miseq PE300 platform. Results are expressed as mean \pm standard deviation of at least ten independent experiments. $\mathrm{P}<0.05$ was considered to indicate a statistically significant difference.

\section{Results}

Patient characteristics and adverse events. The characteristics of the patients are displayed in Table I. No significant difference was observed regarding age, gender or tumor stage between the two groups, and no $C$. butyricum infection was observed following administration of the interventional drug. The difference in body weight, grade of gastrointestinal reaction, hematological indicators and intestinal flora composition were compared between the two groups. At baseline no difference was observed between the two groups regarding weight (Table II). At week 3, a slight weight decrease was observed in the two groups, but the difference was not significant. The severity of nausea and vomiting was lower in the CB group compared with the placebo group. The incidence of diarrhea in the CB group was significantly lower compared with the placebo group (Table III). These results suggested that administration of $C$. butyricum was safe and may reduce the
Table III. Incidence of chemotherapy inducing nausea, vomiting and diarrhea, in the $\mathrm{CB}$ and placebo groups.

\begin{tabular}{|c|c|c|c|}
\hline \multirow[b]{2}{*}{ Adverse effects } & \multicolumn{2}{|c|}{ Group } & \multirow[b]{2}{*}{ P-value } \\
\hline & $\mathrm{CB}$ & Placebo & \\
\hline \multicolumn{4}{|l|}{ Nausea } \\
\hline Grade 1 & $12(60 \%)$ & $8(38.10 \%)$ & 0.166 \\
\hline Grade 2 & $8(40 \%)$ & $13(61.90 \%)$ & \\
\hline Grade 3 & 0 & 0 & \\
\hline \multicolumn{4}{|l|}{ Vomiting } \\
\hline Grade 1 & $3(15 \%)$ & $2(9.52 \%)$ & 0.254 \\
\hline Grade 2 & $15(75 \%)$ & $14(66.67 \%)$ & \\
\hline Grade 3 & $2(10 \%)$ & $5(23.81 \%)$ & \\
\hline Grade 4 & 0 & 0 & \\
\hline Grade 5 & 0 & 0 & \\
\hline \multicolumn{4}{|l|}{ Diarrhea } \\
\hline Absent & $15(75 \%)$ & $8(38.09 \%)$ & 0.017 \\
\hline Grade 1 & $4(20 \%)$ & $9(42.86 \%)$ & \\
\hline Grade 2 & $1(5 \%)$ & $3(14.29 \%)$ & \\
\hline Grade 3 & 0 & 1 & \\
\hline Grade 4 & 0 & 0 & \\
\hline Grade 5 & 0 & 0 & \\
\hline
\end{tabular}

Adverse effects were graded according to the Criteria for Adverse Events. The grade of nausea was divided into three levels. The grades of vomiting and diarrhea were divided into five levels. The incidences between two groups were compared by Wilcoxon test. CB, Clostridium butyricum.

incidence of chemotherapy-induced diarrhea, with little effect on patient weight.

Blood test index. The effects of $C$. butyricum on the inflammatory response, immune function and nutritional status were evaluated by analyzing the differences between the two groups 

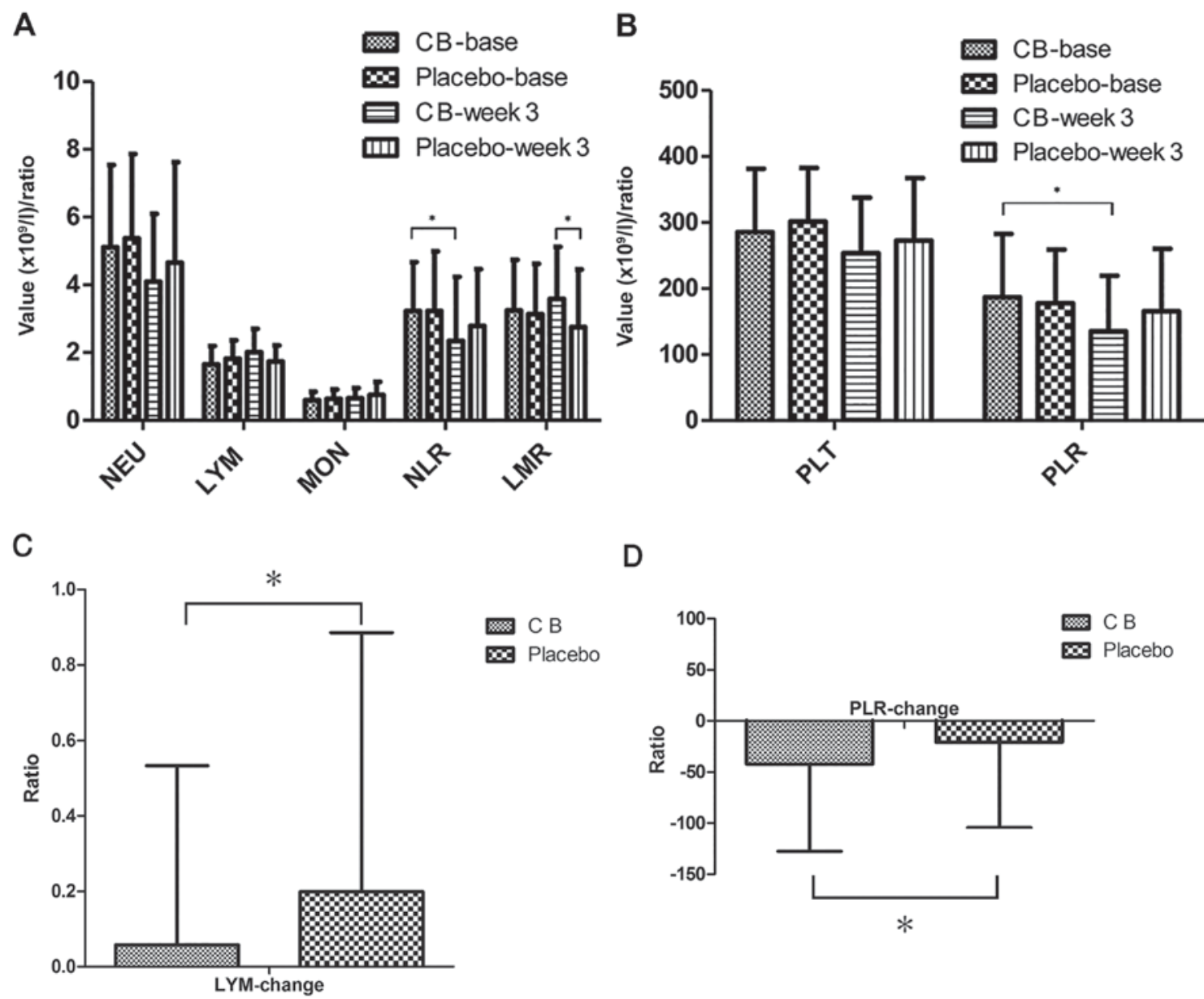

D

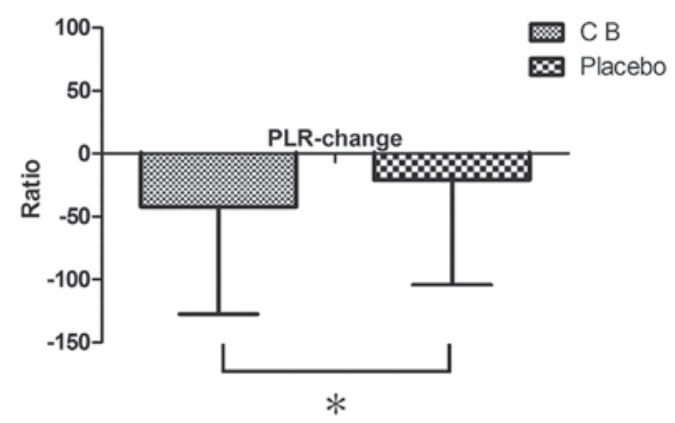

Figure 2. Peripheral blood routine and systemic inflammatory response indicators. (A) Significant differences were identified in LYM between the two groups. NLR decreased significantly in the CB group. At week 3, LMR was significantly higher in the CB group compared the placebo group. (B) PLR decreased significantly in the CB group. (C) The changes of LYM between two groups demonstated significant difference. (D) The changes of PLR between two groups demonstrated significant difference. NEU, neutrophil; LYM, lymphocyte; MON, monocyte; PLT, platelet; NLR, neutrophil lymphocyte ratio; PLR, platelet/lymphocyte ratio; LMR, lymphocyte/monocyte ratio; CB, Clostridium butyricum. ${ }^{*} \mathrm{P}<0.05$.

regarding blood cell count, systemic inflammatory response (SIR) biomarkers, lymphocyte subsets, immunoglobulin and albumin. The change in blood cell count and SIR biomarkers LMR, NLR and PLR was compared (Fig. 2). At baseline, the blood cell count and SIR biomarkers revealed no significant difference between the two groups. At week 3, the number of lymphocytes increased in the $\mathrm{CB}$ group and decreased in the placebo group, and the change between the two groups was significantly different $(\mathrm{P}<0.05)$. PLR decreased in the two groups, but a significant difference was only observed for the CB group. In addition, a significant difference was revealed between the two groups $(\mathrm{P}<0.05)$. NLR decreased in the two groups, but only demonstrated a significant decrease in the $\mathrm{CB}$ group $(\mathrm{P}<0.05)$. LMR increased in the $\mathrm{CB}$ group and decreased in the placebo group, though the difference was not significant. However, at week 3, LMR was significantly higher in the $\mathrm{CB}$ group compared with the placebo group $(\mathrm{P}<0.05)$. Subsequently, the peripheral blood lymphocyte subsets representing the cellular immune system were analyzed (Table IV). Cluster of differentiation (CD) $16^{+} \mathrm{CD} 56^{+}$designation represented the natural killer (NK) cells, and CD19+designation represented the $\mathrm{B}$ lymphocyte population. The results revealed that the levels of $\mathrm{CD}^{+} \mathrm{CD}^{+}$and $\mathrm{CD} 16^{+} \mathrm{CD} 56^{+}$in the $\mathrm{CB}$ group were higher compared with the placebo group, though the difference was not significant. $\mathrm{CD} 3{ }^{+} \mathrm{CD} 4^{+}$percentage and ratio in the $\mathrm{CB}$ group displayed a decreasing trend. However, no significant difference was observed in the changes of these indicators between the two groups. Humoral immune function was also investigated by evaluating $\operatorname{IgG}, \operatorname{IgA}, \operatorname{IgM}$ and $\operatorname{IgE}$ levels (Table V). The results revealed that all of these immunoglobulins decreased in the two groups, although without any significant difference. Alterations in albumin expression levels were also evaluated (Table II). Albumin expression increased in the $\mathrm{CB}$ group and decreased in the placebo group, although the difference was not significant. Overall, through blood indicator analysis, it was established that the SIR index in the CB group was significantly improved compared with the placebo group, suggesting that $C$. butyricum reduces the systemic inflammatory response. However, lymphocyte subsets, immunoglobulin and albumin expression levels did not significantly alter.

Fecal flora analysis. 16S rRNA pyrosequencing was used to analyze variations in fecal flora to evaluate the effects of C. butyricum on intestinal flora. A total of 2,990,797 reads were analyzed following sequence denoising, trimming and chimera picking. The average sequence length was 
Table IV. Percentages of lymphocyte subsets in the CB and placebo groups.

\begin{tabular}{|c|c|c|c|c|c|}
\hline \multirow[b]{2}{*}{ Cell type } & \multirow[b]{2}{*}{ Groups } & \multicolumn{2}{|c|}{ Time point } & \multirow[b]{2}{*}{ P-intra value } & \multirow[b]{2}{*}{ P-value } \\
\hline & & Baseline & Week3 & & \\
\hline T lymphocyte & $\mathrm{CB}$ & $69.62 \pm 10.27$ & $69.94 \pm 11.25$ & 0.9246 & 0.8136 \\
\hline $\mathrm{CD}^{+}$ & $\begin{array}{l}\text { Placebo } \\
\text { P-inter value }\end{array}$ & $\begin{array}{c}70.07 \pm 6.825 \\
0.5919\end{array}$ & $\begin{array}{c}71.20 \pm 9.492 \\
0.7052\end{array}$ & $\begin{array}{c}0.5886 \\
-\end{array}$ & \\
\hline $\mathrm{CD}^{+}{ }^{+} \mathrm{T}$ lymphocyte & $\mathrm{CB}$ & $42.19 \pm 8.329$ & $39.10 \pm 6.848$ & 0.3147 & 0.4984 \\
\hline $\mathrm{CD}^{+}{ }^{+} \mathrm{CD} 4^{+}$ & $\begin{array}{l}\text { Placebo } \\
\text { P-inter value }\end{array}$ & $\begin{array}{c}39.54 \pm 10.13 \\
0.4053\end{array}$ & $\begin{array}{c}41.05 \pm 9.565 \\
0.8923\end{array}$ & 0.8264 & \\
\hline $\mathrm{CD}^{+} \mathrm{T}$ lymphocyte & $\mathrm{CB}$ & $25.87 \pm 8.74$ & $26.81 \pm 8.40$ & 0.7306 & 0.9622 \\
\hline $\mathrm{CD}^{+}{ }^{+} \mathrm{CD} 8^{+}$ & $\begin{array}{l}\text { Placebo } \\
\text { P-inter value }\end{array}$ & $\begin{array}{c}27.13 \pm 8.33 \\
0.6486\end{array}$ & $\begin{array}{c}27.62 \pm 9.82 \\
0.7991\end{array}$ & $\begin{array}{c}0.9281 \\
-\end{array}$ & \\
\hline $\mathrm{CD}^{+} / \mathrm{CD}^{+}$ratio & $\begin{array}{l}\text { CB } \\
\text { Placebo } \\
\text { P-inter value }\end{array}$ & $\begin{array}{c}1.829 \pm 0.6937 \\
1.687 \pm 0.8696 \\
0.5734\end{array}$ & $\begin{array}{c}1.629 \pm 0.5387 \\
1.757 \pm 0.8488 \\
0.5987\end{array}$ & $\begin{array}{c}0.4956 \\
0.8347 \\
-\end{array}$ & 0.6931 \\
\hline Natural killer cell & $\mathrm{CB}$ & $17.70 \pm 8.739$ & $19.41 \pm 11.99$ & 0.4248 & 0.4402 \\
\hline $\mathrm{CD} 16^{+} \mathrm{CD} 56^{+}$ & $\begin{array}{l}\text { Placebo } \\
\text { P-inter value }\end{array}$ & $\begin{array}{c}16.97 \pm 7.214 \\
0.7888\end{array}$ & $\begin{array}{c}17.27 \pm 7.663 \\
0.4262\end{array}$ & $\begin{array}{c}0.8813 \\
-\end{array}$ & \\
\hline $\begin{array}{l}\text { B lymphocyte } \\
\text { CD } 19^{+}\end{array}$ & $\begin{array}{l}\text { CB } \\
\text { Placebo } \\
\text { P-inter value }\end{array}$ & $\begin{array}{c}11.48 \pm 3.689 \\
11.94 \pm 5.349 \\
0.7888\end{array}$ & $\begin{array}{c}9.231 \pm 2.929 \\
10.66 \pm 5.858 \\
0.5567\end{array}$ & $\begin{array}{c}0.0706 \\
0.4007 \\
-\end{array}$ & 0.9875 \\
\hline
\end{tabular}

Results are expressed as the mean \pm standard deviation. P-intra values indicate the P-values between two time points within the same group calculated by Wilcoxon test. P-inter values indicate the P-value between the two groups at the same time point, calculated by Wilcoxon test. $\mathrm{P}$-values were obtained via the comparison of the changes of two groups calculated using the Wilcoxon test. CB, Clostridium butyricum; $\mathrm{CD}$, cluster of differentiation.

435.52 bps. A total of 673 OTUs were clustered by $97 \%$ similarity. The sequencing depth was assessed by plotting the rarefaction curve for each sample (Fig. 3). The majority of the samples reached their plateaus, confirming the adequacy of sequencing. The microbial alpha-diversity between the $\mathrm{CB}$ and placebo groups was compared using the Shannon and Chao indexes (Fig. 4). The difference in diversity between the $\mathrm{CB}$ and the placebo groups were compared at and between the two time points selected. The results indicated that the Shannon and Chao indexes increased in the CB group, yet decreased in the placebo group, although without any indication of significance. $\beta$-diversity was subsequently compared by PCoA analysis. The analysis of OTU levels suggested that the factors explaining the differences in the data were small at baseline or week 3 (Fig. 5). Adonis and ANOSIM analysis were performed, which indicated that grouping did not make sense at baseline or week 3 (Tables VI and VII). These results suggested a minimal difference between the two groups at baseline or week 3. Variation at the phylum (Fig. 6) and genus (Fig. 7) levels were calculated in the two groups. The flora were divided into four phyla: Firmicutes, Bacteroidetes, Proteobacteria and Actinobacteria. The proportion of each phylum in the CB group was marginally altered after 3 weeks, whilst the proportion of Firmicutes decreased significantly in the placebo group $(\mathrm{P}<0.05)$. In regard to the genus level, no significant difference was observed in either group, but specific trends were observed. Beneficial genera including Lactobacillus, and the majority of genera producing short-chain fatty acids, including Faecalibacterium, Clostridium, Blautia and Roseburia, were elevated in the CB group, while the pathogenic bacteria Escherichia-Shigella were decreased. These trends were in contrast with the placebo group. The five most abundant OTUs of the whole flora were Faecalibacterium, Megamonas, Prevotella, Escherichia/Shigella and Bacteroides. The OTU difference between the two groups was compared, and it was revealed that 18 OTUs altered in a significant manner. The first five are displayed in Table VIII. The beneficial OTU Lactobacillus and Clostridium, and the symbiotic OTU Enterobacteriaceae, were significantly increased in the CB group, though decreased in the placebo group. C. butyricum administration resulted in minor effects on the diversity of gut flora. In regard the phylum level, the proportion of each phylum in CB group was marginally altered, while in the placebo group the proportion of Firmicutes decreased significantly. In reference to the genus level, the CB group indicated an advantageous trend toward increased beneficial bacteria, while the pathogenic bacteria were decreased; this difference was not significant. The placebo group indicated the opposite. The beneficial OTUs were significantly increased in the $C B$ group $(P<0.05)$ and decreased in the placebo group $(\mathrm{P}<0.05)$. 
Table V. Serum Ig concentrations in the CB and placebo groups.

\begin{tabular}{|c|c|c|c|c|c|}
\hline \multirow[b]{2}{*}{ Type } & \multirow[b]{2}{*}{ Groups } & \multicolumn{2}{|c|}{ Time points } & \multirow[b]{2}{*}{ P-intra value } & \multirow[b]{2}{*}{ P-value } \\
\hline & & Baseline & Week 3 & & \\
\hline \multirow[t]{3}{*}{$\operatorname{IgG}(\mathrm{g} / \mathrm{l})$} & $\mathrm{CB}$ & $12.44 \pm 2.584$ & $11.61 \pm 1.767$ & 0.1727 & 0.4274 \\
\hline & Placebo & $10.82 \pm 2.769$ & $10.52 \pm 2.232$ & 0.7459 & \\
\hline & P-inter value & 0.05789 & 0.1207 & - & \\
\hline \multirow[t]{3}{*}{$\operatorname{IgA}(\mathrm{g} / \mathrm{l})$} & $\mathrm{CB}$ & $2.341 \pm 0.8865$ & $2.169 \pm 0.7771$ & 0.4099 & 0.5928 \\
\hline & Placebo & $2.316 \pm 0.8892$ & $2.189 \pm 0.6528$ & 0.7781 & \\
\hline & P-inter value & 0.9568 & 0.7917 & - & \\
\hline \multirow[t]{3}{*}{$\operatorname{IgM}(\mathrm{g} / \mathrm{l})$} & $\mathrm{CB}$ & $1.151 \pm 0.3478$ & $1.131 \pm 0.3060$ & 0.9584 & 0.8147 \\
\hline & Placebo & $1.087 \pm 0.4358$ & $1.012 \pm 0.3830$ & 0.8436 & \\
\hline & P-inter value & 0.9451 & 0.8734 & - & \\
\hline \multirow[t]{3}{*}{$\operatorname{IgE}(\mathrm{IU} / \mathrm{ml})$} & $\mathrm{CB}$ & $206.5 \pm 343.0$ & $84.98 \pm 87.45$ & 0.4527 & 0.7162 \\
\hline & Placebo & $133.5 \pm 186.2$ & $99.71 \pm 156.2$ & 0.8436 & \\
\hline & P-inter value & 0.4634 & 0.4753 & - & \\
\hline
\end{tabular}

Results are expressed as the mean \pm standard deviation. P-intra values indicate the P-values between two time points within the same group calculated by Wilcoxon test. P-inter values indicate the P-value between the two groups at the same time point, calculated by Wilcoxon test. P-values were obtained via the comparison of the changes of two groups calculated using the Wilcoxon test. CB, Clostridium butyricum; Ig, immunoglobulin.

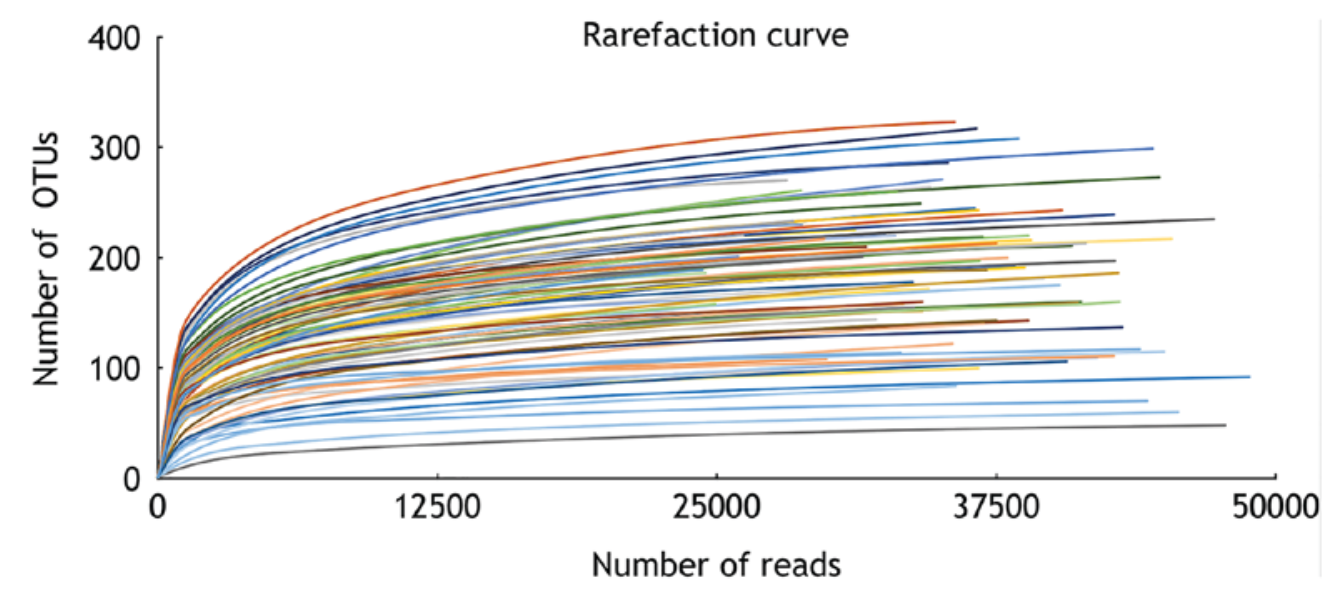

Figure 3. Rarefaction curve. Curves tended to plateau as the number of sequencing events increased, suggesting that the samples were completely sequenced. OTU, operational taxonomy unit.
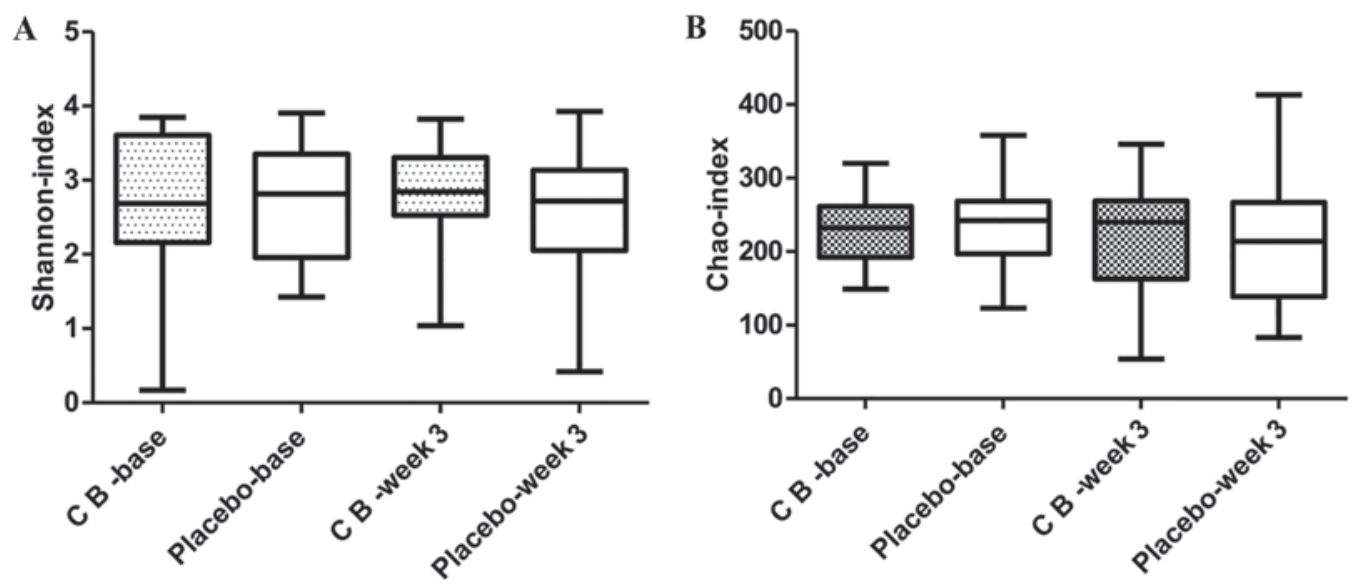

Figure 4. Diversity indexes. (A) Shannon and (B) Chao indexes. The differences between groups and time points were compared, and no significant differences were indicated. The change between groups was also not significantly different. CB, Clostridium butyricum. 

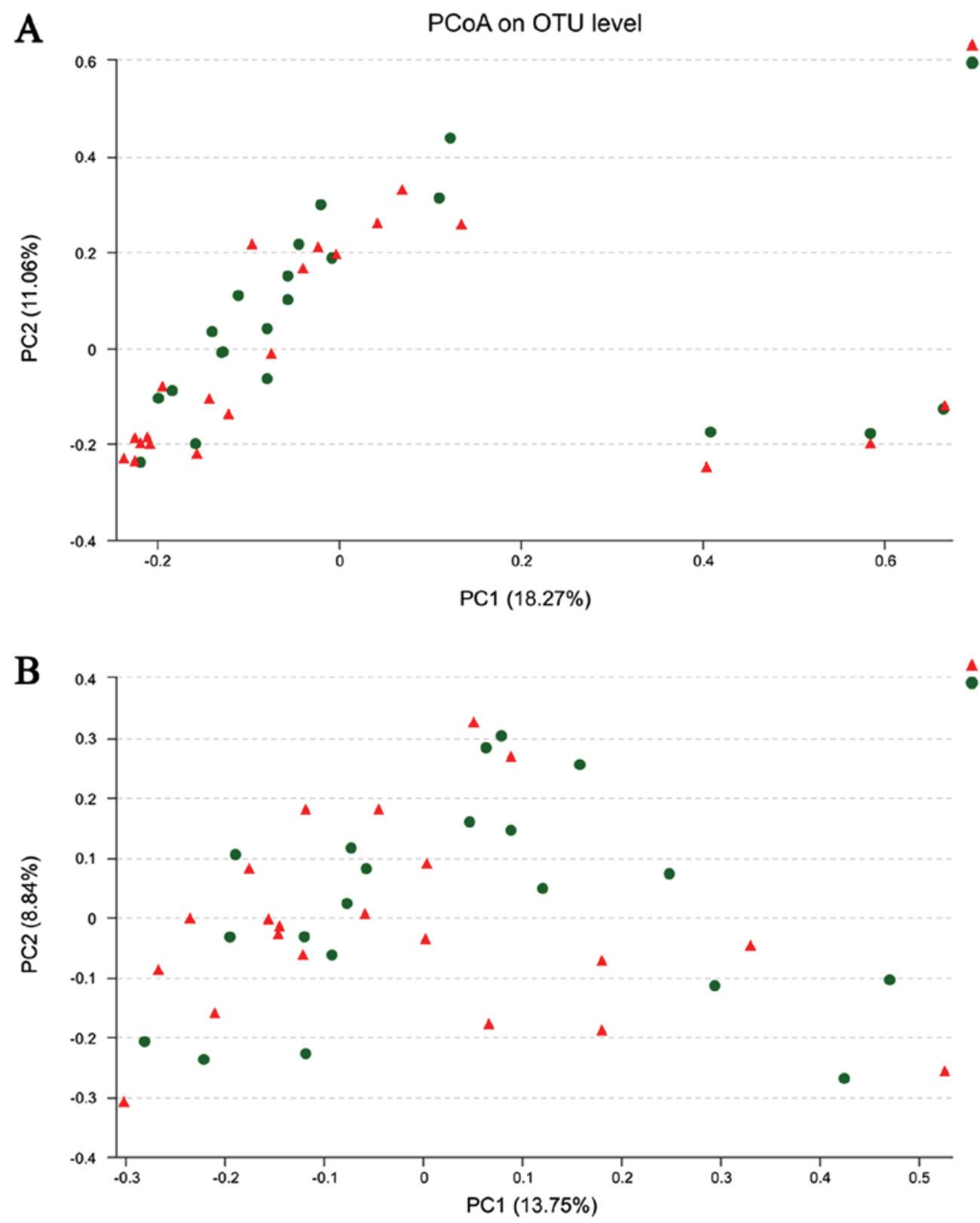

Figure 5. PCoA analysis. PCoA analysis on OTU levels at (A) baseline and (B) week 3. PC1 and PC2 indicates the two most important factors impacting the grouping. No significant impact was identified to the grouping at the two time points. T1: CB group at baseline; T2: CB group at week 3. C1: placebo group at baseline; $\mathrm{C} 2$ : placebo group at week 3. CB, Clostridium butyricum; PCoA, principal coordinates analysis; OTU, operational taxonomy unit. PC, principal coordinates; $\mathrm{C}$, control; $\mathrm{T}$, treatment.

\section{Discussion}

The present study was prospective, random, double blind and placebo comparative, providing an indication that C. butyricum administration was a safe and effective treatment for chemotherapy-induced diarrhea in patients with lung cancer. C. butyricum maintained gut homeostasis and alleviated inflammatory response. However, no clear effects were obtained regarding immunity or nutrition.

Probiotics have been used in numerous types of gastrointestinal diseases, due to their ability to preserve the stability of the intestinal microenvironment. $C$. butyricum is a spore-like micro-ecological preparation. It was selected primarily for its notable tolerance to the gastric environment and temperature. C. butyricum produces butyric acid, the preferred energy source for intestinal epithelial cells, and promotes the proliferation of Bifidobacteria and Lactobacilli (24). It is also able to regulate gut homeostasis and alleviate the inflammatory response in IBD (15). Therefore, it was speculated that a potential beneficial effect against chemotherapy-induced diarrhea may be associated with numerous factors, including intestinal epithelial apoptosis, intestinal barrier dysfunction, intestinal flora structural changes and proinflammatory cytokine production (12). The results revealed that $C$. butyricum administration to patients with lung cancer reduced the incidence of chemotherapy-induced diarrhea. This result was similar to those obtained in earlier studies (25-28). The basis for this may involve intestinal epithelial cell repair promotion, intestinal homeostasis maintenance and intestinal inflammation reduction (29). 
Table VI. ANOSIM analysis between the two groups of the operational taxonomy unit level at baseline and week 3 .

\begin{tabular}{lccc}
\hline ANOSIM & Statistic & P-value & $\begin{array}{c}\text { Permutation } \\
\text { number }\end{array}$ \\
\hline Baseline & -0.0154 & 0.69 & 999 \\
Week 3 & -0.0315 & 0.905 & 999 \\
\hline
\end{tabular}

ASOSIM, analysis of similarity.

The diversity of the intestinal microflora ensures the stability of the intestinal microecology (30). Chemotherapy usually leads to reduced diversity of the intestinal flora $(31,32)$; a previous study highlighted a marked increase in Bacteroides and Escherichia following chemotherapy and a corresponding decrease in Blautia, Faecalibacterium and Roseburia at the genus level (33). In the present study, the diversity of the gut flora in the CB group did not decrease following chemotherapy, and the proportion of each phylum altered slightly, while in the placebo group, the proportion of Firmicutes decreased significantly. These results suggested that $C$. butyricum maintained the stability of the intestinal microflora. Furthermore, levels of such pathogenic bacteria as Bacteroides and Escherichia were decreased in the CB group, while beneficial bacteria, including Blautia, Faecalibacterium and Roseburia increased in number. These results may suggest that $C$. butyricum aided in the maintenance of gut homeostasis, and may also be responsible for the lower incidence of chemotherapy-induced diarrhea in the CB group.

Short-chain fatty acids (SCFAs) are considered to have beneficial effects, including nourishing intestinal epithelial cells and energy components to protect the intestinal mucosal barrier, reducing the level of inflammation and enhancing gastrointestinal motility (34). A previous study (35) revealed that SCFA-producing genera including Phascolarctobacterium, Roseburia, Blautia, Faecalibacterium, Clostridium, Subdoligranulum, Ruminococcus, Coprococcus and Bacteroides form the bulk of the gut flora to maintain human health; C. butyricum produces butyric acid, an SCFA, in the intestine. In the present study it was established that the majority of these genera were elevated in the $\mathrm{CB}$ group, suggesting that $C$. butyricum may promote the growth of SCFA-producing gut flora, thus facilitating intestinal mucosal repair and decreasing the incidence of diarrhea.

Cancer has been described as the "wound that does not heal' (36). Thus, cancer is a course of persistent wounding and healing, with characteristic inflammation. Cells of the myeloid and lymphoid lineage have different effects on cancer progression and metastasis (37-39). Neutrophil degranulation of chemically toxic products and growth factors may have important effects on cancer progression, angiogenesis and invasion (40-43). Peripheral monocytes are recruited in tumor tissue by various chemokines (44). They may not only stimulate angiogenesis and enhance tumor cell invasion, but also prevent anti-tumor immunoreaction by natural killer and T cells during tumor progression and following chemotherapy $(45,46)$. Platelets secrete cytokines and growth factors which may contribute to cancer progression by influencing angiogenesis, cell migration, proliferation and epithelial to mesenchymal cell transition (47-49). Furthermore, lymphocytes serve a critical role in tumor defense by inducing cytotoxic cell death, and may suppress tumor cell proliferation and invasion $(50,51)$. The results of the present study have demonstrated that neutrophils, monocytes and platelets serve important roles in promoting inflammation and cancer, while lymphocytes may suppress tumor formation. Therefore, the ratio of the number of neutrophils, monocytes and platelets to the number of lymphocytes, (i.e. SIR biomarkers, including NLR, PLR and LMR) may serve as a reference for the degree of systemic inflammation in patients with cancer. Previous studies (52-54) illustrated that SIR biomarkers are associated with the progression and prognosis of lung cancer; increased NLR and PLR, and decreased LMR are associated with poor prognosis in lung cancer. The present study indicated that SIR biomarkers were significantly increased in the CB group compared with the placebo group, suggesting that $C$. butyricum effectively reduced the systemic inflammatory response.

It was hypothesized that $C$. butyricum may initially act by reducing intestinal inflammation. Within 3 weeks of administration $C$. butyricum may reduce the inflammatory response without causing notable variations in the intestinal flora. Increased incubation times or doses may have altered the composition of the gut flora, although this speculation requires further investigation.

Fluctuationsinimmunefunction and nutritional status were also investigated following administration of C. butyricum. Other studies have demonstrated that probiotics may inhibit the proliferation and growth of tumor cells $(55,56)$, which may be associated with enhanced immunity. Probiotics interact with intestinal epithelial cells through a variety of pattern recognition receptors, affecting cellular immune function and the inflammatory response (57-61). They are also able to stimulate the humoral immune response and increase $\operatorname{IgG}$ and $\operatorname{IgA}$ levels in a number of diseases (62-65). Few studies are available regarding probiotics and cellular immune function in patients with cancer. Zhang et al (66) performed a study on the effects of C. butyricum and Bifidobacterium on lymphocyte differentiation in late preterm infants, which revealed increased $\mathrm{CD}^{+}$and decreased $\mathrm{CD}^{+} \mathrm{T}$ cell numbers in the probiotic group compared with the control group (treated with simple formula milk); although the proportions of NK and B were not significantly different between the two groups. By contrast, the present study suggested an increased number of $\mathrm{CD} 8^{+} \mathrm{T}$ cells and NK cells, and a decreased number of $\mathrm{CD}^{+} \mathrm{T}$ cells in the $\mathrm{CB}$ group, although no significant difference was observed, suggesting that $C$. butyricum may promote the proliferation of $\mathrm{CD} 8^{+} \mathrm{T}$ cells and NK cells, to a cytotoxic end. It was speculated that these effects may be beneficial in patients with lung cancer. Further studies have illustrated that probiotics increase the concentration of $\operatorname{IgG}$ and $\operatorname{IgA}$ in patients with colorectal cancer, following surgery $(67,68)$. The present study illustrated that the concentration of all Igs decreased in the two groups, and the alterations were not statistically different. Therefore, it was supposed that $C$. butyricum administration for 3 weeks may not have a significant effect on the humoral immune response of patients undergoing chemotherapy. 
Table VII. Adonis analysis between the two groups of the operational taxonomy level at baseline and week 3.

\begin{tabular}{llrcccrr}
\hline Time point & \multicolumn{1}{c}{ ADONIS } & Df & Sums of Sqs & Mean Sqs & F.Model & $\mathrm{R}^{2}$ & $\operatorname{Pr}(>\mathrm{F})$ \\
\hline Baseline & Group_factor\$G & 1 & 0.2563 & 0.25630 & 0.73394 & 0.01847 & 0.796 \\
& Residuals & 39 & 13.6191 & 0.34921 & 0.98153 & & \\
& Total & 40 & 13.8754 & 1.00000 & & & 0.963 \\
Week 3 & Group_factor\$G & 1 & 0.1901 & 0.19006 & 0.61786 & 0.0156 & \\
& Residuals & 39 & 11.9971 & 0.30762 & 0.9844 & & \\
& Total & 40 & 12.1872 & 1.00000 & & & \\
& &
\end{tabular}

Group_factor\$G, grouping scheme; Df, degree of freedom; SumsOfSqs, total variance; MeanSqs, mean square (difference); F.Model, F test value; $\mathrm{R}^{2}$, reflects grouping differences, the higher the value, the higher the grouping differences; Pr, P-value $>0.05$ indicating the high degree of reliability of this test.
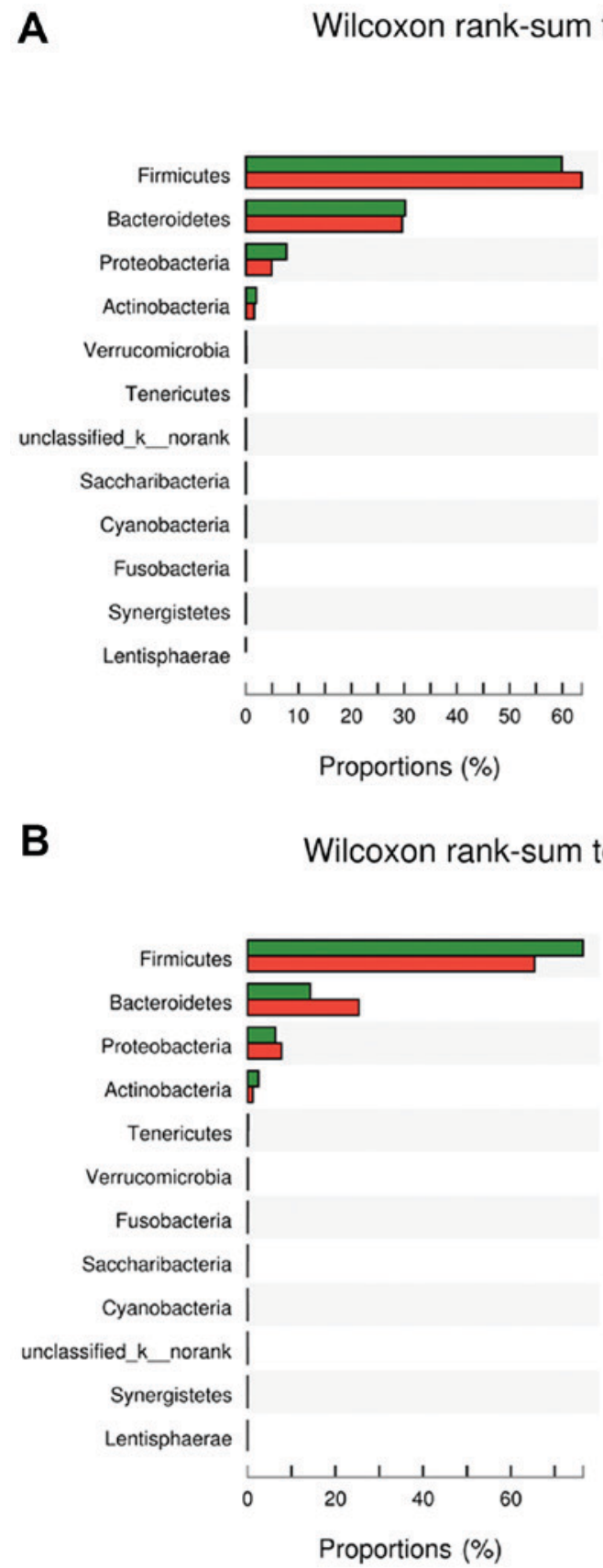

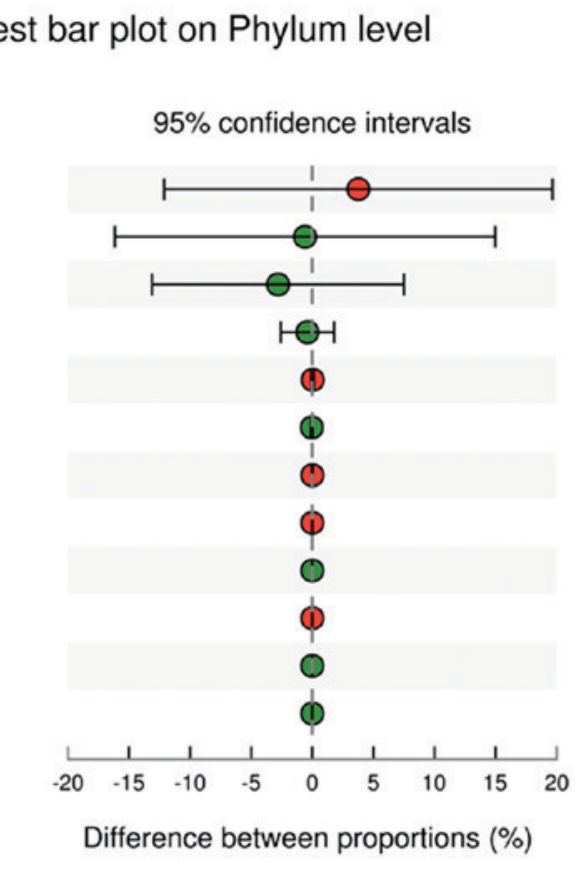

0.6359

0.9031

0.525

0.4903

0.6926

0.148

0.7898

0.4522

0.4629

0.7596

0.8617

0.1626

\section{bar plot on Phylum level}

C1

C2

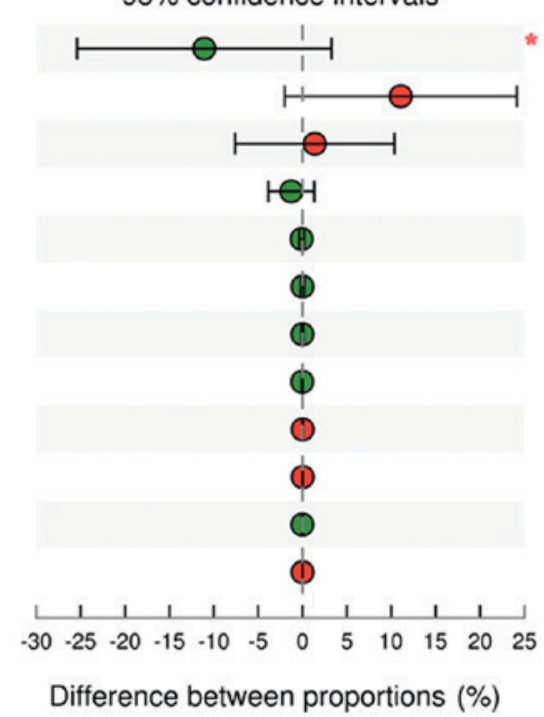

0.03913

0.07826

0.4504

0.8405

0.1213

0.4269

0.4193

0.9899

0.6244

0.3836

0.3481

0.9803

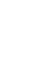

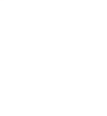




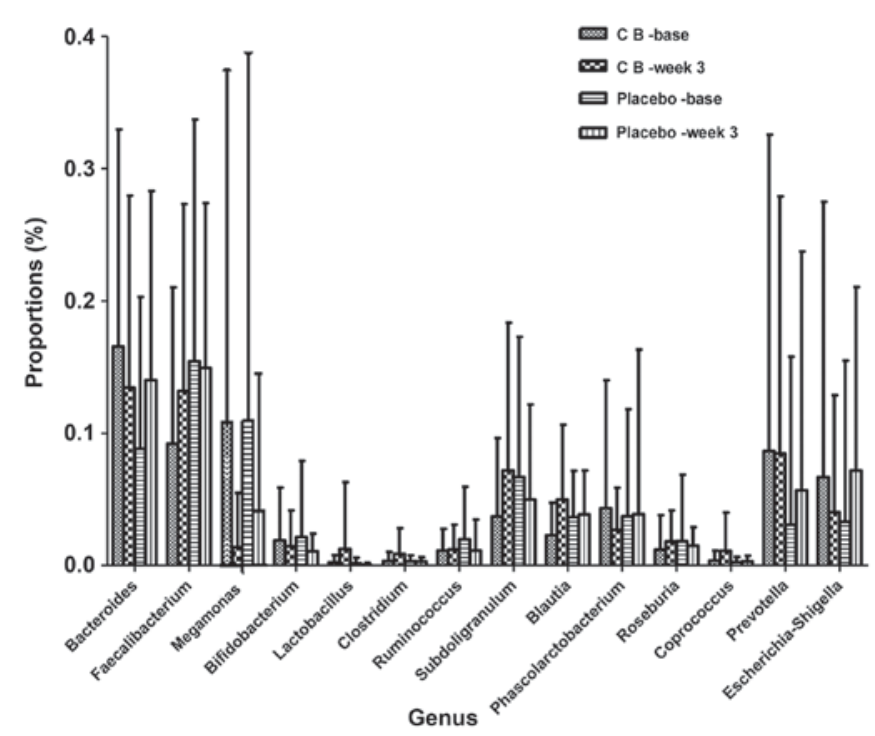

Figure 7. Genus differences in the $\mathrm{CB}$ and placebo groups. No significant difference was observed in either group. However, the most beneficial genera including Faecalibacterium, Lactobacillus, Clostridum, Blautia and Roseburia were elevated in number. The pathogenic bacteria Escherichia/Shigella decreased in the CB group, and increased in the placebo group. CB, Clostridium butyricum.

Due to the rapid proliferation and consumption of nutrients by cancer cells, patients with malignant tumors are prone to malnutrition and cachexia. Albumin is the most abundant protein in serum and, to a certain extent, reflects the nutritional status of patients. Studies have demonstrated that supplementation with probiotics may improve the nutritional status of mice, increase serum albumin and maintain weight $(25,69)$. The present study did not reveal a significant difference in serum albumin or weight in the CB group, although in placebo group, there was a decrease in albumin and weight. These results indicated that $C$. butyricum administration for 3 weeks may not significantly impact the nutritional status of patients with cancer.

The present study had the following limitations: i) The number of participants was small, and the participants were not from multiple centers; ii) the intervention dose and time may be insufficient, thus specific indicators including immune function, nutritional status and fluctuations in flora genus did not significantly alter; and iii) due to the complex interplay between the intestinal flora and the human body, the study did not exclude confounding factors, including diet and drugs.

In conclusion, the present study indicated that supplementation with the probiotic $C$. butyricum in patients with lung cancer is safe. It decreased chemotherapy-induced diarrhea, reduced the systemic inflammatory response, and helped to maintain the condition of the intestinal flora. However, C. butyricum also weakly influenced the immune and nutrition status of patients. Despite this, the present results provided important evidence for probiotic supplementation during chemotherapy.

\section{Acknowledgements}

The authors would like to thank Dr Zie Wang and her colleagues (Department of Oncology, Shandong Provincial 
Hospital Affiliated to Shandong University) for their help with the laboratory blood testing. The authors would like to thank Dr Yan Zhang (Department of Gastroenterology, Shandong University, Qilu Hospital) for his advice regarding the figures and manuscript.

\section{Funding}

The present study was supported by the National Natural Science Foundation of China (grant no. 81700457 and 81670489).

\section{Availability of data and materials}

The datasets used and/or analyzed during the current study are available from the corresponding author on reasonable request.

\section{Authors' contributions}

YT, ML and YQL designed the study; YT performed the research, with the assistance of RJ and WS. YT and ML analyzed the data. YT generated the figures and wrote the paper. ML revised the figures and critically revised the manuscript for important intellectual content.

\section{Ethics approval and consent to participate}

All participants provided written informed consent. The protocols were approved by the Institutional Ethics Committee of Shandong Provincial Hospital (ClinicalTrials.gov identifier, NCT02771470) and conducted in accordance with the approved guidelines.

\section{Patient consent for publication}

Not applicable.

\section{Competing interests}

The authors declare that they have no competing interests.

\section{References}

1. Chen W, Zheng R, Baade PD, Zhang S, Zeng H, Bray F, Jemal A, Yu XQ and He J: Cancer statistics in China, 2015. CA Cancer J Clin 66: 115-132, 2016.

2. Hirsch FR, Scagliotti GV, Mulshine JL, Kwon R, Curran WJ Jr, Wu YL and Paz-Ares L: Lung cancer: Current therapies and new targeted treatments. Lancet 389: 299-311, 2017.

3. Howington JA, Blum MG, Chang AC, Balekian AA and Murthy SC: Treatment of stage I and II non-small cell lung cancer: Diagnosis and management of lung cancer, 3rd ed: American College of Chest Physicians evidence-based clinical practice guidelines. Chest 143 (5 Suppl): e278S-e313S, 2013.

4. Johnson DH, Schiller JH and Bunn PA Jr: Recent clinical advances in lung cancer management. J Clin Oncol 32: 973-982, 2014.

5. Wakelee H, Kelly K and Edelman MJ: 50 Years of progress in the systemic therapy of non-small cell lung cancer. Am Soc Clin Oncol Educ Book 2014: 177-189, 2014.

6. Bradbury P, Sivajohanathan D, Chan A, Kulkarni S, Ung Y and Ellis PM: Postoperative adjuvant systemic therapy in completely resected non-small-cell lung cancer: A systematic review. Clin Lung Cancer 18: 259-273.e8, 2017.

7. Andreyev J, Ross P, Donnellan C, Lennan E, Leonard P, Waters C, Wedlake L, Bridgewater J, Glynne-Jones R, Allum W, et al: Guidance on the management of diarrhoea during cancer chemotherapy. Lancet Oncol 15: e447-e460, 2014.
8. Marx W, Kiss N, McCarthy AL, McKavanagh D and Isenring L: Chemotherapy-induced nausea and vomiting: A narrative review to inform dietetics practice. J Acad Nutr Diet 116: 819-827, 2016.

9. Tong $\mathrm{H}$, Isenring $\mathrm{E}$ and Yates $\mathrm{P}$ : The prevalence of nutrition impact symptoms and their relationship to quality of life and clinical outcomes in medical oncology patients. Support Care Cancer 17: 83-90, 2009.

10. Tohyama N, Tanaka S, Onda K, Sugiyama K and Hirano T: Influence of anticancer agents on cell survival, proliferation, and CD4+CD25+Foxp3+ regulatory T cell-frequency in human peripheral-blood mononuclear cells activated by $\mathrm{T}$ cell-mitogen. Int Immunopharmacol 15: 160-166, 2013.

11. Verma R, Foster RE, Horgan K, Mounsey K, Nixon H, Smalle N, Hughes TA and Carter CR: Lymphocyte depletion and repopulation after chemotherapy for primary breast cancer. Breast Cancer Res 18: 10, 2016.

12. Touchefeu Y, Montassier E, Nieman K, Gastinne T, Potel G, Bruley des Varannes S, Le Vacon F and de La Cochetière MF: Systematic review: The role of the gut microbiota in chemotherapy- or radiation-induced gastrointestinal mucositis-current evidence and potential clinical applications. Aliment Pharmacol Ther 40: 409-421, 2014.

13. Reid G; Food and Agricultural Organization of the United Nation and the WHO: The importance of guidelines in the development and application of probiotics. Curr Pharm Des 11: 11-16, 2005.

14. Jonkers D, Penders J, Masclee A and Pierik M: Probiotics in the management of inflammatory bowel disease: A systematic review of intervention studies in adult patients. Drugs 72: 803-823, 2012.

15. Kanai T, Mikami Y and Hayashi A: A breakthrough in probiotics: Clostridium butyricum regulates gut homeostasis and anti-inflammatory response in inflammatory bowel disease. J Gastroenterol 50: 928-939, 2015.

16. Seki H, Shiohara M, Matsumura T, Miyagawa N, Tanaka M, Komiyama A and Kurata S: Prevention of antibiotic-associated diarrhea in children by Clostridium butyricum MIYAIRI. Pediatr Int 45: 86-90, 2003.

17. Yasueda A, Mizushima T, Nezu R, Sumi R, Tanaka M, Nishimura J, Kai Y, Hirota M, Osawa H, Nakajima K, et al: The effect of Clostridium butyricum MIYAIRI on the prevention of pouchitis and alteration of the microbiota profile in patients with ulcerative colitis. Surg Today 46: 939-949, 2016.

18. Gibson RJ, Keefe DM, Lalla RV, Bateman E, Blijlevens N, Fijlstra M, King EE, Stringer AM, van der Velden WJ, Yazbeck R, et al: Systematic review of agents for the management of gastrointestinal mucositis in cancer patients. Support Care Cancer 21: 313-326, 2013.

19. Mego M, Holec V, Drgona L, Hainova K, Ciernikova S and Zajac V: Probiotic bacteria in cancer patients undergoing chemotherapy and radiation therapy. Complement Ther Med 21: 712-723, 2013.

20. Wood DE, Kazerooni E, Baum SL, Dransfield MT, Eapen GA, Ettinger DS, Hou L, Jackman DM, Klippenstein D, Kumar R, et al: Lung cancer screening, version 1.2015: Featured updates to the NCCN guidelines. J Natl Compr Canc Netw 13: 23-34; quiz 34, 2015.

21. Chen AP, Setser A, Anadkat MJ, Cotliar J, Olsen EA, Garden BC and Lacouture ME: Grading dermatologic adverse events of cancer treatments: The common terminology criteria for adverse events version 4.0. J Am Acad Dermatol 67: 1025-1039, 2012.

22. Hills LP and Tiffany TO: Comparison of turbidimetric and light-scattering measurements of immunoglobulins by use of a centrifugal analyzer with absorbance and fluorescence/light-scattering optics. Clin Chem 26: 1459-1466, 1980.

23. Quast C, Pruesse E, Yilmaz P, Gerken J, Schweer T, Yarza P, Peplies J and Glöckner FO: The SILVA ribosomal RNA gene database project: Improved data processing and web-based tools. Nucleic Acids Res 41 (Database Issue): D590-D596, 2013.

24. Kong Q, He GQ, Jia JL, Zhu QL and Ruan H: Oral administration of Clostridium butyricum for modulating gastrointestinal microflora in mice. Curr Microbiol 62: 512-517, 2011.

25. Yeh KY, Wang HM, Chang JW, Huang JS, Lai CH, Lan YJ, Wu TH, Chang PH, Wang H, Wu CJ, et al: Omega-3 fatty acid-, micronutrient-, and probiotic-enriched nutrition helps body weight stabilization in head and neck cancer cachexia. Oral Surg Oral Med Oral Pathol Oral Radiol 116: 41-48, 2013.

26. Lee JY, Chu SH, Jeon JY, Lee MK, Park JH, Lee DC, Lee JW and Kim NK: Effects of 12 weeks of probiotic supplementation on quality of life in colorectal cancer survivors: A double-blind, randomized, placebo-controlled trial. Dig Liver Dis 46: 1126-1132, 2014. 
27. Sadahiro S, Suzuki T, Tanaka A, Okada K, Kamata H, Ozaki T and Koga Y: Comparison between oral antibiotics and probiotics as bowel preparation for elective colon cancer surgery to prevent infection: Prospective randomized trial. Surgery 155: 493-503, 2014

28. Mego M, Chovanec J, Vochyanova-Andrezalova I, Konkolovsky P, Mikulova M, Reckova M, Miskovska V, Bystricky B, Beniak J, Medvecova L, et al: Prevention of irinotecan induced diarrhea by probiotics: A randomized double blind, placebo controlled pilot study. Complement Ther Med 23: 356-362, 2015.

29. Zhang HL, Yu LX, Yang W, Tang L, Lin Y, Wu H, Zhai B, Tan YX, Shan L, Liu Q, et al: Profound impact of gut homeostasis on chemically-induced pro-tumorigenic inflammation and hepatocarcinogenesis in rats. J Hepatol 57: 803-812, 2012.

30. Zheng J, Gänzle MG, Lin XB, Ruan L and Sun M: Diversity and dynamics of bacteriocins from human microbiome. Environ Microbiol 17: 2133-2143,2015.

31. Fijlstra M, Ferdous M, Koning AM, Rings EH, Harmsen HJ and Tissing WJ: Substantial decreases in the number and diversity of microbiota during chemotherapy-induced gastrointestinal mucositis in a rat model. Support Care Cancer 23: 1513-1522, 2015.

32. Xu X and Zhang X: Effects of cyclophosphamide on immune system and gut microbiota in mice. Microbiol Res 171: 97-106, 2015.

33. Montassier E, Batard E, Massart S, Gastinne T, Carton T, Caillon J, Le Fresne S, Caroff N, Hardouin JB, Moreau P, et al: $16 \mathrm{~S}$ rRNA gene pyrosequencing reveals shift in patient faecal microbiota during high-dose chemotherapy as conditioning regimen for bone marrow transplantation. Microb Ecol 67: 690-699, 2014

34. Canani RB, Costanzo MD, Leone L, Pedata M, Meli R and Calignano A: Potential beneficial effects of butyrate in intestinal and extraintestinal diseases. World J Gastroenterol 17: 1519-1528, 2011.

35. Wang T, Cai G, Qiu Y, Fei N, Zhang M, Pang X, Jia W, Cai S and Zhao L: Structural segregation of gut microbiota between colorectal cancer patients and healthy volunteers. ISME J 6 : 320-329, 2012

36. Dvorak HF: Tumors: Wounds that do not heal. Similarities between tumor stroma generation and wound healing. N Engl J Med 315: 1650-1659, 1986

37. DeNardo DG, Andreu P and Coussens LM: Interactions between lymphocytes and myeloid cells regulate pro-versus anti-tumor immunity. Cancer Metastasis Rev 29: 309-316, 2010.

38. Donskov F: Immunomonitoring and prognostic relevance of neutrophils in clinical trials. Semin Cancer Biol 23: 200-207, 2013.

39. Caronni N, Savino B and Bonecchi R: Myeloid cells in cancer-related inflammation. Immunobiology 220: 249-253, 2015.

40. Sandhu JK, Privora HF, Wenckebach G and Birnboim HC: Neutrophils, nitric oxide synthase, and mutations in the mutatect murine tumor model. Am J Pathol 156: 509-518, 2000.

41. Imai Y, Kubota Y, Yamamoto S, Tsuji K, Shimatani M, Shibatani N, Takamido S, Matsushita M and Okazaki K: Neutrophils enhance invasion activity of human cholangiocellular carcinoma and hepatocellular carcinoma cells: An in vitro study. J Gastroenterol Hepatol 20: 287-293, 2005.

42. Dumitru CA, Lang S and Brandau S: Modulation of neutrophil granulocytes in the tumor microenvironment: Mechanisms and consequences for tumor progression. Semin Cancer Biol 23 141-148, 2013.

43. Powell DR and Huttenlocher A: Neutrophils in the tumor microenvironment. Trends Immunol 37: 41-52, 2016.

44. Siveen KS and Kuttan G: Role of macrophages in tumour progression. Immunol Lett 123: 97-102, 2009.

45. Gordon S and Martinez FO: Alternative activation of macrophages: Mechanism and functions. Immunity 32: 593-604, 2010

46. Noy R and Pollard JW: Tumor-associated macrophages: From mechanisms to therapy. Immunity 41: 49-61, 2014

47. Banks RE, Forbes MA, Kinsey SE, Stanley A, Ingham E, Walters $\mathrm{C}$ and Selby PJ: Release of the angiogenic cytokine vascular endothelial growth factor (VEGF) from platelets: Significance for VEGF measurements and cancer biology. Br J Cancer 77: 956-964, 1998.

48. Labelle M, Begum S and Hynes RO: Direct signaling between platelets and cancer cells induces an epithelial-mesenchymal-like transition and promotes metastasis. Cancer Cell 20: 576-590, 2011.

49. Peterson JE, Zurakowski D, Italiano JE Jr, Michel LV, Connors S, Oenick M, D'Amato RJ, Klement GL and Folkman J: VEGF, PF4 and PDGF are elevated in platelets of colorectal cancer patients. Angiogenesis 15: 265-273, 2012.
50. Dunn GP, Bruce AT, Ikeda H, Old LJ and Schreiber RD: Cancer immunoediting: From immunosurveillance to tumor escape. Nat Immunol 3: 991-998, 2002.

51. Grivennikov SI, Greten FR and Karin M: Immunity, inflammation, and cancer. Cell 140: 883-899, 2010.

52. Cedrés S, Torrejon D, Martínez A, Martinez P, Navarro A, Zamora E, Mulet-Margalef $\mathrm{N}$ and Felip E: Neutrophil to lymphocyte ratio (NLR) as an indicator of poor prognosis in stage IV non-small cell lung cancer. Clin Transl Oncol 14: 864-869, 2012

53. Sánchez-Lara K, Turcott JG, Juárez E, Guevara P, Núñez-Valencia C, Oñate-Ocaña LF, Flores D and Arrieta O: Association of nutrition parameters including bioelectrical impedance and systemic inflammatory response with quality of life and prognosis in patients with advanced non-small-cell lung cancer: A prospective study. Nutr Cancer 64: 526-534, 2012.

54. Käsmann L, Bolm L, Schild SE, Janssen S and Rades D: Neutrophil-to-lymphocyte ratio predicts outcome in limited disease small-cell lung cancer. Lung 195: 217-224, 2017.

55. Sivan A, Corrales L, Hubert N, Williams JB Aquino-Michaels K, Earley ZM, Benyamin FW, Lei YM, Jabri B, Alegre ML, et al: Commensal bifidobacterium promotes antitumor immunity and facilitates anti-PD-L1 efficacy. Science 350: 1084-1089, 2015.

56. Alexander JL, Wilson ID, Teare J, Marchesi JR, Nicholson JK and Kinross JM: Gut microbiota modulation of chemotherapy efficacy and toxicity. Nat Rev Gastroenterol Hepatol 14: 356-365, 2017.

57. Sanz Y and De Palma G: Gut microbiota and probiotics in modulation of epithelium and gut-associated lymphoid tissue function. Int Rev Immunol 28: 397-413, 2009.

58. Hardy H, Harris J, Lyon E, Beal J and Foey AD: Probiotics, prebiotics and immunomodulation of gut mucosal defences: Homeostasis and immunopathology. Nutrients 5: 1869-1912, 2013.

59. Ashraf R and Shah NP: Immune system stimulation by probiotic microorganisms. Crit Rev Food Sci Nutr 54: 938-956, 2014.

60. Abdollahi-Roodsaz S, Abramson SB and Scher JU: The metabolic role of the gut microbiota in health and rheumatic disease: Mechanisms and interventions. Nat Rev Rheumatol 12: 446-455, 2016.

61. Dwivedi M, Kumar P, Laddha NC and Kemp EH: Induction of regulatory T cells: A role for probiotics and prebiotics to suppress autoimmunity. Autoimmun Rev 15: 379-392, 2016.

62. Sindhu KN, Sowmyanarayanan TV, Paul A, Babji S, Ajjampur SS, Priyadarshini S, Sarkar R, Balasubramanian KA, Wanke CA, Ward HD and Kang G: Immune response and intestinal permeability in children with acute gastroenteritis treated with Lactobacillus rhamnosus GG: A randomized, double-blind, placebo-controlled trial. Clin Infect Dis 58: 1107-1115, 2014.

63. Mansouri-Tehrani HA, Rabbani-Khorasgani M, Hosseini SM, Mokarian F, Mahdavi H and Roayaei M: Effect of supplements: Probiotics and probiotic plus honey on blood cell counts and serum IgA in patients receiving pelvic radiotherapy. J Res Med Sci 20: 679-683, 2015.

64. Gagnon M, Vimont A, Darveau A, Fliss I and Jean J: Study of the ability of bifidobacteria of human origin to prevent and treat rotavirus infection using colonic cell and mouse models. PLoS One 11: e0164512, 2016

65. Karamese M, Aydin H, Sengul E, Gelen V, Sevim C, Ustek D and Karakus E: The immunostimulatory effect of lactic acid bacteria in a rat model. Iran J Immunol 13: 220-228, 2016.

66. Zhang SF, Tang ZS, Tong L, Tao XX, Suo QF and Xu XM: Effects of clostridium butyricum and bifidobacterium on BTLA expression on CD4+ T cells and lymphocyte differentiation in late preterm infants. Microb Pathog 100: 112-118, 2016.

67. Zhang JW, Du P, Gao J, Yang BR, Fang WJ and Ying CM: Preoperative probiotics decrease postoperative infectious complications of colorectal cancer. Am J Med Sci 343: 199-205, 2012.

68. Zhu D, Chen X, Wu J, Ju Y, Feng J, Lu G, Ouyang M, Ren B and $\mathrm{Li} Y$ : Effect of perioperative intestinal probiotics on intestinal flora and immune function in patients with colorectal cancer. Nan Fang Yi Ke Da Xue Xue Bao 32: 1190-1193, 2012 (In Chinese).

69. Bowen JM, Stringer AM, Gibson RJ, Yeoh AS, Hannam S and Keefe DM: VSL\#3 probiotic treatment reduces chemotherapy-induced diarrhea and weight loss. Cancer Biol Ther 6 : 1449-1454, 2007

This work is licensed under a Creative Commons Attribution-NonCommercial-NoDerivatives 4.0 International (CC BY-NC-ND 4.0) License. 